\title{
Analysis and application research of new type online monitoring technologies for substation equipment
}

\author{
Rong Cheng ${ }^{1, *}$, Linjie Chai ${ }^{1}$, Shiyao $\mathrm{Hu}^{1}$ and Yongpeng Shen ${ }^{1}$ \\ ${ }^{1}$ State Grid Hebei Economic Research Institute, Shijiazhuang, Hebei, 050000, China
}

\begin{abstract}
At present, a variety of online monitoring technologies for substation equipment including winding temperature by optical fiber, however, the accuracy and stability show great differences among them. This paper focuses on the advancement, feasibility and economics of various new technologies, combined with the construction and operation experience of wisdom substations, in order to select mature, reliable, and effective new online monitoring technologies that reduce the amount of equipment operation and maintenance from a number of new technologies. In the end, the article gives suggestions on the application of various new online monitoring technologies.
\end{abstract}

\section{Background}

Online monitoring technology can perceive the status of primary and secondary equipment in the substation by different principles, which detect faulty equipment, improve the operation and maintenance efficiency of the substation, and ensure the safety of the power grid. At present, on-line monitoring technologies such as transformer oil chromatogram and iron core ground current have become very common and become a key method for pre-judgment and diagnosis of the equipment in substations. For example, tens of thousands of transformer oil chromatogram online monitoring devices have been installed in the State Grid Corporation of China. These devices have detected the defects of the main equipment thousands of times and have been proved to be quite effective and reliable for detecting latent faults inside the oil-immersed transformer.

Recently, the SGCC has built a number of wisdom substations, applying more than a dozen new online monitoring technologies, including transformer winding temperature by optical fiber, partial discharge of transformer, water in SF6 of gas insulated switchgear(GIS). The maturity, accuracy, and maintenance of these new technologies are in different levels. Some new technologies have advanced principles and high equipment failure detection rates. However, there are also some new technologies have very high requirements on manufacturing levels, and high false alarm rates. Based on this, this article selects mature, reliable, and effective new online monitoring technologies that reduce the amount of equipment operation and maintenance from a number of new technologies, based on the construction of wisdom substations, comprehensively considering many factors such as technology maturity, monitoring accuracy, practical application effects, investment benefits. A new type of panoramic perception mode of substation equipment is construct in this paper, which play the role of the online monitoring devices to ensure the safety and stable operation of the main equipment in substations.

\section{Analysis of online monitoring technologies}

\subsection{Online monitoring technology of the transformer}

\subsubsection{Maintenance-free online monitoring technology of transformer oil chromatogram}

There have been tens of thousands of online monitoring devices of transformer oil chromatogram are operated in the power grid. Most of them serve 220KV transformers. The feature gases generated in the event of a transformer malfunction include $\mathrm{H} 2, \mathrm{CO}, \mathrm{CO} 2, \mathrm{CH} 4, \mathrm{C} 2 \mathrm{H} 2, \mathrm{C} 2 \mathrm{H} 4$, and $\mathrm{C} 2 \mathrm{H} 6$. Different malfunctions of transformers produce different feature gas. The oil chromatographic devices analyse the gas in insulating oil to test early fault inside the equipment. After years of operation, the device has obtained mature operation and service experience.

At present, the oil chromatographic online monitoring device based on gas chromatographic prototype is widely used. It generally uses a carrier bottle, and each bottle can be used 400 times (approximately two months). Due to frequently replacement, the devices cost a lot of operation and maintenance costs.

Maintenance-free online monitoring technology of transformer oil chromatogram use air as a raw material and air compressor as power. It removes moisture, carbon dioxide, microplate impurities, and interference gases in air by water gas separation, drying, purification,

\footnotetext{
* Corresponding author's e-mail::chengrong1991@126.com
} 
etc., and finally obtain dry and clean carrier gas to achieve maintenance-free.

The technology increases investment about RMB 80,000 per transformer, but it can greatly reduce maintenance work, which save operation and maintenance costs. Considering the device has been trial running in multiple substations and has not been faulty, and relevant technical specifications are issued, it is recommended to promote the application of this technology in this paper.

\subsubsection{Online monitoring technology of transformer winding temperature by optical fiber}

Currently, the winding temperature of the transformer is inferred by a thermometer measuring the top oil temperature, which can not accurately reflect the actual temperature of the winding. The online monitoring technology of transformer winding temperature by optical fiber realize distributed measurement of winding temperature through optical fiber put together with the coil during production, which can accurately measure the winding temperature. This technology determines the buried position of the optical fiber sensor based on the cloud image calculated by the thermal field and install point type optical fiber sensor or string type sensor on the high voltage winding of the transformer, as shown in figure 1 . The sensors transmit the signal to the background through the internal optical fiber, external optical fiber, and modem to form a temperature distribution curve to determine the temperature of the transformer winding. The online monitoring technology of transformer winding temperature by optical fiber has high measurement accuracy, can detect defects and hidden dangers in time, which is of great significance for improving the safety and stable operation of the transformer.

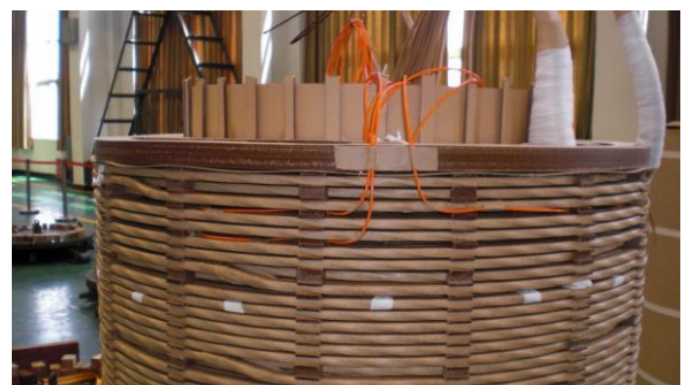

Figure 1. Schematic of embedding the temperature sensor by optical fiber

Due to the fragility of the optical fiber and its probe is easily contaminated by dust, this technology has very high requirements on the manufacturing process, and problems are likely to occur after the equipment is installed such as fiber is break. According to the current relevant data, the life of the optical fiber sensor is about 30 years, does not match the life of the transformer of 60 years. Therefore, online monitoring technology of transformer winding temperature by optical fiber has not been promoted by the SGCC and CSPG.

Applying this technology on a $220 \mathrm{kV}$ transformer will increase investment by RMB 230,000, while RMB 160,000 of a $110 \mathrm{kV}$ transformer. Considering the investment and maturity, it is recommended to first apply the technology on $110 \mathrm{kV}$ transformers with a low capacity-load ratio, and promote the application of this technology after accumulating enough operating experience.

\subsubsection{Online monitoring technology of transformer winding deformation by optical fiber}

Currently, the winding deformation of the transformer can only obtained by regular test between power outage overhaul or test after equipment malfunction. Online monitoring technology of transformer winding deformation by optical fiber build the pressure sensor by optical fiber in the pressure shoe position of the oilimmersed transformer, and transmit the signal to the background through the internal optical fiber, external optical fiber, and modem to realize the measurement of transformer winding deformation (compression force), as shown in figure 2.
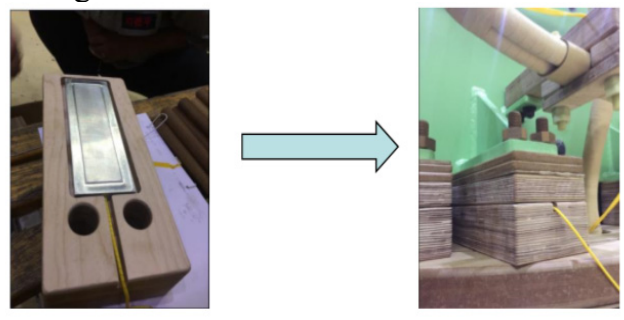

Figure 2. Diagram of installing the pressure sensor by optical fiber

At the same with online monitoring technology of transformer winding temperature by optical fiber, this technology is still immature and lacks operation experience. Currently, there is no relevant technical specifications and acceptance standards. Based on the above description, this paper recommends the suspension of the application of the technology.

\subsubsection{Online monitoring technology of transformer partial discharge}

Partial discharge is both a precursor for transformer insulation aging, and it is also an important reason for insulation breakdown. Many faults of the transformer can be reflected in the change of quantity and mode of partial discharge. Conventional substation lacks monitoring methods for the partial discharge of transformers. Online monitoring technology of transformer partial discharge can track changes of the device's insulation state, determine the location of partial discharge by using the special high frequency signal principle. Transformers are accompanied by electrical pulses, electromagnetic radiation, ultrasonic waves during partial discharge. The special high frequency method has advantages of strong anti-interference ability, high sensitivity, and can identify different insulation defects. $70 \%$ of the online monitoring devices of transformer partial discharge use special high frequency method.

At present, the error report rate, communication malfunction is common in the operation monitoring 
devices. Combining that, the transformer has a lower chance to make partial discharge and the technique needs to open holes in the transformer body increasing the leak dangers of the transformer, the technology is not recommended promotion in this paper.

\subsection{Online monitoring technology of GIS}

\subsubsection{Online monitoring technology of GIS partial discharge}

In substations with high voltage levels and complex environments, the deterioration of equipment insulation will have a huge impact on the safe operation of the power grid. Especially in the case of the application of GIS equipment, due to the large number of gas chambers and low visibility, it is particularly important to monitor the internal partial discharge of the GIS equipment. In addition, the operation department has an urgent need for partial discharge monitoring, and it is hoped that the maintenance strategy can be adjusted in time according to the trend of defects.

At present, part of the $220 \mathrm{kV}$ GIS in conventional substations are combined with built-in partial discharge sensors, which does not have the function of data transmission. When inspections need performed, operation staffs need to carry the terminal to the substation, and then export the data for calculation. Online monitoring technology of GIS partial discharge can continuously detect, and track changes in the insulation status of the equipment in time.

The technology is mature, and each monitoring point of the $220 \mathrm{kV}$ GIS will increase investment about RMB 20,000 and RMB 10,000 for $110 \mathrm{kV}$ GIS. In addition, the relevant technical specifications have been promulgated, and equipment manufacturers can comply with them. Therefore, this article recommends the promotion and application of this technology in $110 \mathrm{kV}$ and above GIS to strengthen the monitoring of the partial discharge of GIS equipment and discover malfunctions in time.

\subsubsection{Online monitoring technology of water in SF6 of GIS}

The GIS equipment is full of SF6 gas, if the content of water in the SF6 gas exceeds the standard, the electrical performance of the GIS equipment will be greatly affected. At present, it mainly relies on the test to obtain water content in the GIS equipment during a power outage, and there is no online continuous monitoring. Online monitoring technology of water in SF6 of GIS add water density transmitters for continuous monitoring.

For the GIS equipment is under positive pressure, it is difficult for water to enter from the outside and diffuse inside. However, the sensors is generally installed in the corner of the equipment, as shown in figure 3 , and the moisture is more difficult to reach, so the measured value of the online monitoring device cannot reflect the actual situation of the internal water. If the device generates a false alarm, it will increase the workload of the operation stuff instead.
The investment of one point of online monitoring device of water in SF6 of GIS is about RMB 10,000. Coupled with the above reasons, it is not recommended to use this technology for testing at the moment.
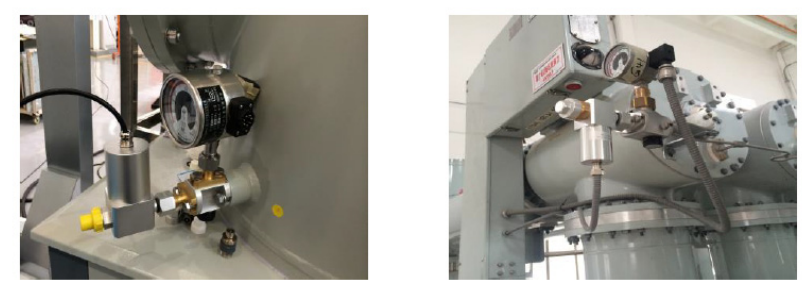

Figure 3. Diagram of online monitoring device of water in SF6 of GIS

\subsubsection{Online monitoring technology of breaker mechanical characteristics of GIS}

At present, it mainly relies on the test to obtain breaker mechanical characteristics in the GIS equipment during a power outage. Online monitoring technology of breaker mechanical characteristics of GIS can measure and record parameters such as the stroke curve of the moving contact, the current waveform of the opening and closing operating coil, the current waveform of the energy storage motor, and the status of the auxiliary contact switch after the breaker is activated. This technology can determine the operating status of the mechanism and realize the non-stop monitoring of the mechanical characteristics of the circuit breaker.

This system needs to install a variety of different types of sensors in different parts of the breaker. The accuracy and reliability of the sensor needs to be further verified. When the sensor has accuracy problems or operating failures, it will cause data errors and false alarms. In addition, the monitoring system can only test when the breaker is acting. Since the breaker has few actions under normal conditions, there are few opportunities to collect characteristic parameters for the system. Therefore, the online monitoring system is difficult to make accurate judgments with few data. Therefore, this technology is still not recommended in high voltage equipment such as $110 \mathrm{kV}$ GIS and above.

\subsection{Online monitoring technology of switchgear}

\subsubsection{Online monitoring technology of contact temperature in switchgear}

The high-voltage switchgear of the substation is an important electrical equipment, during long-term operation, the contacts of the breaker in the switchgear will heat up due to aging or excessive contact resistance. Conventional substation switchgear use infrared temperature measurement, which can only measure the surface temperature of the cabinet, and it is difficult to detect the heating of the components inside. If the hot parts cannot be monitored in time and take measures, it will eventually lead to a fire accident.

Online monitoring technology of contact temperature in switchgear adopts self-powered wireless temperature 
measurement technology to realize the real-time monitoring of the temperature of the breaker plum blossom contact finger, bus bar lap surface, and outlet cable head lap. The technology is mature, 435 sets of devices have been installed in many substations such as Wenzhang and Liangzhuang. At present, the devices are running well, and the investment is relatively reasonable, about RMB 2,000 per switchgear. Therefore, it is recommended to promote the application of this technology.

\subsubsection{Online monitoring technology of breaker mechanical characteristics of switchgear}

The mechanical characteristics of switchgear breakers in conventional substations are mainly obtained through tests between power outage. Online monitoring technology of breaker mechanical characteristics of switchgear can record data such as the stroke curve of the moving contact, the current waveform of the opening and closing operating coil, the current waveform of the energy storage motor, and the status of the auxiliary contact switch after the breaker is activated. After the data is uploaded to the monitoring host, the expert diagnosis system analyzes and judges the operation status of the organization and finds hidden dangers.

Taking into account the low probability of mechanical failure of the equipment, combined with economic efficiency, about RMB 235,00 per switchgear, it is recommended to use it in the reactive circuit of more frequent switching.

\subsubsection{Online monitoring technology of switchgear partial discharge}

Partial discharge may occur between the conductive parts and insulating parts inside the high-voltage switchgear, resulting in a decrease in electrical insulation. If the technology is mature and reliable, online monitoring technology of switchgear partial discharge can find faults and hidden dangers to ensure the stable operation of the switchgear.

According to various discharge phenomena generated in the process of partial discharge, the commonly used partial discharge detection methods for high-voltage switchgear include ERA (pulse current method), ultrasonic (AE) detection method, optical measurement method, chemical detection method, infrared detection method, and radio frequency detection. Any single detection method for partial discharge in switchgear has limitations. Among them, the pulse current detection method and the ultrasonic detection method have poor anti-interference ability, and the UHF detection method, as a non-contact detection method, also has the problem of external noise interference. Compared with GIS partial discharge, because there are more electrical equipment and more cable interfaces in the switchgear, resulting interference signals are also more, which will affect the results of monitoring. So, this article recommends that the technology be applied on a pilot basis first, and then promoted after the accuracy is improved
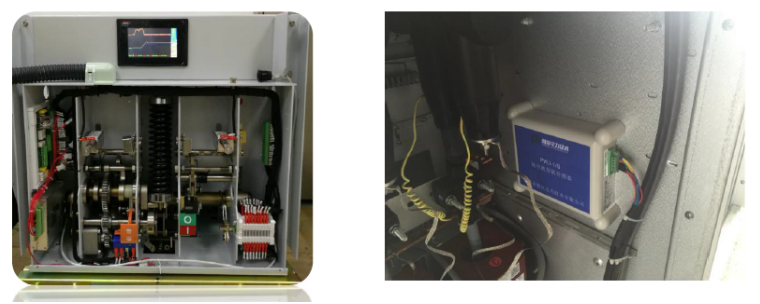

Figure 4. Diagram of online monitoring devices of switchgear partial discharge

\section{Conclusion}

Through the above comparative analysis of the advancement, feasibility and economy of each new technology, we can get the following conclusions, as shown in Table 1.

Table 1. conclusion of every online monitoring technology

\begin{tabular}{|c|c|c|}
\hline Equipment & Monitoring content & Recommendation \\
\hline \multirow{4}{*}{$\begin{array}{c}\text { transforme } \\
\mathrm{r}\end{array}$} & oil chromatogram & $\begin{array}{l}\text { promote the } \\
\text { application }\end{array}$ \\
\hline & winding temperature & promote cautiously \\
\hline & winding deformation & promote cautiously \\
\hline & partial discharge & promote cautiously \\
\hline \multirow{3}{*}{ GIS } & partial discharge & $\begin{array}{l}\text { promote the } \\
\text { application }\end{array}$ \\
\hline & water in SF6 & do not promote \\
\hline & $\begin{array}{c}\text { mechanical } \\
\text { characteristics }\end{array}$ & $\begin{array}{l}\text { promote when } \\
\text { conditions permit }\end{array}$ \\
\hline \multirow{3}{*}{ switchgear } & contact temperature & $\begin{array}{l}\text { promote the } \\
\text { application }\end{array}$ \\
\hline & $\begin{array}{c}\text { mechanical } \\
\text { characteristics }\end{array}$ & $\begin{array}{c}\text { promote in reactive } \\
\text { circuits }\end{array}$ \\
\hline & partial discharge & promote cautiously \\
\hline
\end{tabular}

As shown in the table above, in the transformer online monitoring technologies, oil chromatogram is promoted in this paper, while winding temperature, winding deformation and partial discharge are promoted cautiously. In the GIS online monitoring technologies, partial discharge is promoted in this paper, while water in SF6 is not promoted and mechanical characteristics is promoted when conditions permit. In the switchgear online monitoring technologies, contact temperature is promoted in this paper, while mechanical characteristics is promoted in reactive circuits and partial discharge is promoted cautiously.

\section{References}

1. Dengming Xiao, Zhousheng Zhang, Yibin Huang. (2009) Investigation of the present situation and prospects of the transformer online monitoring. High-Technology \& Industrialization, 5: 103-105

2. Kangning $\mathrm{Xu}$, Kejun Zhang. (2014) The development trend of transformer online monitoring technology. Electric Safety Technology, 16: 64-67

3. Hua Yu. (2012) The key technology of intelligent transformer online monitoring. KEJI YU QIYE, 23: 301 
4. Ming Wang. (2020) Elementary introduction of application of transformer online monitoring technology. Equipment management and maintenance, 23

5. Jiarui Tian. (2020) Research on application of online monitoring technology for power system substation equipment. Modern industrial economy and informatization, 10 